\title{
Universality in an integer Quantum Hall transition
}

\author{
P.T. Coleridge \\ Institute for Microstructural Sciences, National Research Council, Ottawa, Ontario, K1A OR6, Canada
}

(5 February 1999)

An integer Quantum Hall effect transition is studied in a modulation doped p-SiGe sample. In contrast to most examples of such transitions the longitudinal and Hall conductivities at the critical point are close to 0.5 and $1.5\left(\mathrm{e}^{2} / \mathrm{h}\right)$, the theoretically expected values. This allows the extraction of a scattering parameter, describing both conductivity components, which depends exponentially on filling factor. The strong similarity of this functional form to those observed for transitions into the Hall insulating state and for the $\mathrm{B}=\mathbf{0}$ metal-insulator transition implies a universal quantum critical behaviour for the transitions. The observation of this behaviour in the integer Quantum Hall effect, for this particular sample, is attributed to the short-ranged character of the potential associated with the dominant scatterers.

PACS numbers: 73.40.Hm, 71.30. $+\mathrm{h}, 73.20 . \mathrm{Dx}$

Despite the precision with which Integer Quantum Hall (IQH) effect plateaus are quantised the behaviour between plateaus is not yet well understood. Two recent review articles. 6 have pointed out that for these continuous quantum phase transitions the predictions of theory are only partially confirmed by experiment. Some of these predictions are: a peak value of the conductivity tween IQH states $\sigma_{x x}^{c}$ with a universal value of $\mathrm{e}^{2} / 2 \mathrm{~h}$ 目, temperature scaling behaviour showing a $T^{\kappa}$ dependence with $\kappa$ probably close to $3 / 7$ and the two components $f$ f the conductivity connected by a semi-circular relation 6 . Experimentally, in samples showing the best scaling behaviour values of $\sigma_{x x}^{c}$ are less than $\mathrm{e}^{2} / 2 \mathrm{~h}$ and where values of $\sigma_{x x}^{c}$ are close to 0.5 scaling does not usually appear to worki. For transitions from the high field Hall insulator (HI) phase, into the $\nu=1$ plateau, or the FQHE $\nu=1 / 3$ plateau the sitution is somewhat better. There are now several papers 8 which find transitions that appear to scale well, with the expected critical values for $\sigma_{x x}$ and $\sigma_{x y}$.

Results are presented here for a $\nu=2$ to 1 IQH transition which conform, in many ways, to the theoretical expectations. They are obtained in a strained, modulation doped p-SiGe sample with properties that differ, in a number of ways, from the usual GaAs based heterojunctions. This points to some of the factors that usually suppress the theoretically predicted behaviour. An interpretation of these results is offered which supports the universal aature of the transition.

The sample 10 has a density of $3.4 \times 10^{15} \mathrm{~m}^{-2}$, a transport mobility $\left(\mu_{t r}\right)$ of $1.3 \mathrm{~m}^{2} / \mathrm{Vs}$ and a quantum mobility $\left(\mu_{q}\right)$ of $1.5 \mathrm{~m}^{2} / \mathrm{Vs}$. Although these mobilities appear rather low, the quantum lifetime $\left(\tau_{q}=2.4 \mathrm{ps}\right)$ is in fact equal to that of a GaAs based 2D-electron gas (2DEG) with a mobility over $100 \mathrm{~m}^{2} /$ Vs. This is because in GaAs the effective mass is significantly smaller $\left(.067 \mathrm{~m}_{e}\right.$ compared with $0.27 \mathrm{~m}_{e}$ here) and the ratio $\mu_{t r} / \mu_{q}$ is typically 20 . The value of about one seen here implies the dominant scattering mechanism in p-SiGe samples involves a short-ranged potential.

The holes reside in the $\mathrm{J}=3 / 2,\left|M_{J}\right|=3 / 2$, heavy hole band, split-off from the light hole band by strain and confinement 10 . In low magnetic fields the spin-splitting (or more correctly parity splitting)is large, approximately $70 \%$ of the cyclotron spacing. At higher fields there is a strong exchange enhancement of the splitting so at filling factor $\nu=2$ the $(1 \uparrow)$ and $(0 \downarrow)$ levels have crossed (see figure 1 ) and the system becomes ferromagnetically polarised11. Activation measurements show the spinsplitting at $\nu=2$ is almost twice the cyclotron spacing so the (1个) Landau level, involved in the $\nu=2$ to 1 Quantum Hall transition, is very well removed from adjacent Landau levelst22.

Figure 1 shows $\rho_{x x}$ data at several temperatures for the $\nu=2$ to $1 \mathrm{IQH}$ transition obtained in a $200 \mu \mathrm{m}$ wide Hall bar with a measuring current of $1 \mathrm{nA}$. There is a well defined fixed point on the low field side of the peak and inverting the resistivity data (see figure 2a) shows this point corresponds to $\sigma_{x x}=0.46$ and $\sigma_{x y}=1.52$ (in units of $\mathrm{e}^{2} / \mathrm{h}$ ), close to the expected values of 0.5 and 1.5. Figure 3 shows a scaling plot of the data from figure 1 collapsed onto a single curve by plotting it against $\left(\nu_{c}-\nu\right) / \mathrm{T}^{\kappa}$. This involves no adjustable parameters, the critical value $\nu_{c}(=1.612)$ is determined by the field at the fixed point and the exponent $\kappa(=3 / 7)$ has been taken as the generally accepted theoretical value J $^{\text {. Scal- }}$ ing behaviour is obtained for temperatures between 70 and $180 \mathrm{mK}$, a comparable quality of fit is obtained for $\kappa$ varying by about $10 \%$. At higher temperatures deviations, illustrated by the $400 \mathrm{mK}$ data, occur simultaneously in the peak height, in the peak width, and in the movement of the "fixed point " where the curve crosses the collapsed data. Below $70 \mathrm{mK}$ the width changes little, consistent with an effective temperature limited to about $60 \mathrm{mK}$. It is not clear whether this is a genuine effect or just reflects an experimental inability to cool the carriers significantly below this temperature.

Figure $2 \mathrm{~b}$ shows $\sigma_{x x}$ plotted against $\sigma_{x y}$ with the pre- 
dicted semicircular behaviour 6 shown by a dashed line. There is a general deviation of approximately $10 \%$ between the two curves. The model used in Ref. 4 suggests the conductivity components (for the $\nu=2$ to 1 transition) should be given by

$$
\sigma_{x x}=s /\left(1+s^{2}\right) \quad \sigma_{x y}=2-s^{2} /\left(1+s^{2}\right)
$$

where s, a parameter that varies from 0 to $\infty$ through the transition, is equal to 1 at the critical point. It can be identified with $\sigma_{x x}^{(b)}$ in the Chern-Simons mapping or equivalently with the ratio $\mathrm{R} / \mathrm{T}$ where $\mathrm{R}$ and $\mathrm{T}$ are respectively quantum percolation reflection and transmission coefficients. Figure 4 shows values of s extracted from both $\sigma_{x x}$ and $\sigma_{x y}$ using these expressions. For the data derived from $\sigma_{x y}$ (solid line) $\ln (\mathrm{s}$ ) varies approximately linearly with the filling factor $\delta \nu=\nu_{c}-\nu$. Well away from $\delta \nu=0$ the noise, and maybe some of the deviations from linear behaviour, should be attributed to the fitting procedure whereby $\mathrm{s}^{2}$ is fitted to the small deviations of $\sigma_{x y}$ from quantised plateau values.

For the values derived from $\sigma_{x x}$ (dashed lines) there is a discontinuity around $\delta \nu=0$ because of the small deviation of the peak value of $\sigma_{x x}$ from the exact value of 0.5. Away from $\delta \nu=0$, however, there is a general agreement between the two separately determined values of s. Renormalising the $\sigma_{x x}$ data to the 0.5 at $\delta \nu=0$ produces excellent agreement between the two values of $\mathrm{s}$ near $\delta \nu=0$ without any significant change for larger vbalues of $\delta \nu$. A feature of the $\sigma_{x x}$ results is the very high degree of inversion symmetry. This is demonstrated in fig. 4 , by simultaneously plotting s against $\delta \nu$ and $1 / \mathrm{s}$ against $-\delta \nu$ on the same graph where it can be seen that $s(\delta \nu)=1 / s(-\delta \nu)$ over a wide range of $\mathrm{s}$.

The other important feature of the results in fig.4 is the essentially linear dependence of $\ln (\mathrm{s})$ on $\delta \nu$ around $\delta \nu=0$. Combined with the scaling observed in figure 3 this implies, for small values of $\delta \nu$

$$
s=\exp \left[\left(\nu_{c}-\nu\right)\left(T_{0} / T\right)^{\kappa}\right],
$$

with $T_{0}$ a constant, approximately equal to $280 \mathrm{~K}$. This behaviour is not consistent with the procedure used in Ref. 1 to relate s to $\delta \nu$ but is reminiscent of the model proposed by Dobrosavljevic et al13 to describe the $\mathrm{B}=$ 0 metal- insulator transition in Si-MOSFETs 4 where it explains naturally the inversion symmetry observed between $\rho_{x x}$ and $1 / \rho_{x x}$ on the two sides of the transition 15 . A similar expression, but with a different temperature dependence, has also been proposed by Shahar et al 6 to explain the IQH-Hall Insulator transition in GaAs based samples. In this case the symmetry around $\delta \nu=0$ is observed in the I-V curves 18 as well as in $\rho_{x x}$.

The connection between the IQH 1-2 transition and the IQH-HI transition is seen very clearly if the formalism outlined in eqns 1 and 2 is applied to Hall insulator transition, but with $\sigma_{x y}=1-s^{2} /\left(1+s^{2}\right)$. The resistivities obtained by inverting $\sigma_{x x}$ and $\sigma_{x x}$ are then just $\rho_{x x}=\mathrm{s}$ and $\rho_{x y}=1$. The exponential dependence of $\rho_{x x}$ on $\delta \nu$ found in Ref. 16 is therefore the same as that in Eqn.2 indicating the very direct equivalence between the two transition,19. A very similar equivalence has previously been noted by Shahar et al.17 although without an explicit evaluation of s. Incorporating an E field dependence into Eqn. 2 can also empirically account for the symmetry observed in the I-V curve. In particular for the absence of any $\mathrm{I}-\mathrm{V}$ dependence in the $\rho_{x y}$ data for this transition 18 .

The specific functional dependence of $\mathrm{s}$, indicative of a continuous quantum phase transition, therefore explains both the transition between IQH phases and the IQH-HI transition. Further, if $\mathrm{s}$ is identified with the resistivity at $\mathrm{B}=0$ (and $\delta \nu$ with the density changes) it is also consistent with the behaviour of the $\mathrm{B}=0$ metal-insulator transition 13 . These transitions all therefore appear to belong to the same universality class. There remains, however, the problem of the residual discrepancy of about $10 \%$ between the observed and expected values of $\sigma_{x x}$ and the related question of why, in most samples, the discrepancy is so much larger. These large discepancies are presumeably the reason the intrinsic properties of the phase transition are not always revealed in the transport data.

The p-SiGe system differs from the more common GaAs and GaInAs based systems in a number of ways that may be relevent to this issue. They include: approximately equal values of $\tau_{q}$ and $\tau_{t r}$ indicating a shortranged scattering potential; spin resolved Landau levels that are well separated; a spin polarised system; and an IQH transition occurring in the $N_{L}=1$, not $N_{L}=0$ Landau level.

The data reported in Ref. 17 show a $\nu=2$ to 1 IQHE transition very similar to that reported here. The peak value of $\sigma_{x x}$ is about 0.45 and an approximately semicircular relationship between $\sigma_{x x}$ and $\sigma_{x y}$ is also observed. The sample there was a low mobility GaAs based 2DEG: a value of $\mu_{q}$ is not available but the low mobility, for relatively high density, implies the dominant scatterers are almost certainly impurities in the channel. Unlike remote donor impurities these have a short-ranged scattering potential. The common feature between these two samples is the short-ranged potential. This is therefore, almost certainly, the reason for the similar, almost canonical, behaviour of the quantum Hall transition in both cases.

In high mobility GaAs based 2DEGs, where the scattering potential is long ranged, the ratio $\tau_{t r} / \tau_{q}$ is large because the momentum weighting term $(1-\cos \theta)$ is small. In these circumstances the quantum diffusion model gives quantitatively correct answersen. It predicts peak values of $\sigma_{x x}$ that are reduced by a factor of approximately $\tau_{q} / \tau_{t r}$ from those given for simple $\delta$-function scattering 21 . This ratio represents the difference between the lifetime $\tau_{q}$, that characterises the Landau level width (and hence the peak value of the density of states) and the transport time $\tau_{t r}$ which includes the condition that multiple small angle scattering events are needed to randomise the ve- 
locity of the carriers.

In the same spirit, in IQH transitions when localisation effects are important, although the scaling behaviour may be dominated by the band of itinerant states near the Landau level centre the observed conductivity will also depend on the transport process and the range of the scattering. For example, in quantum percolation models the conductivity is determined by quantum amplitudes that depend on the degree of tunnelling at saddle points 422 . Explicitly it is obtained from these amplitudes by using a one dimensional, edge state, model where there is exact cancellation between the density of states and the velocity $\left(v_{F}\right)$. In practice this model will not always be valid and the transport will remain, to some extent, 2 -dimensional. The component of velocity that determines the conductivity will then take a range of values, not just $\pm v_{F}$. For $\tau_{q} \approx \tau_{t r}$, the exact one-dimensional cancellation between the density of states and the velocity will be approximately reproduced and the conductivity should then reflect quite accurately the behaviour of the quantum amplitudes and the associated universality. Generally, however, there is a "memory" associated with the multiple scattering events that are needed to randomise the carriers when scattered by a long-range potential and this reduces $\sigma_{x x}$ below the "universal" value. This reduction, like that seen in the quantum diffusion model, depends on the range of the potential and will be sample dependent.

The calculations in Ref. 3 also give universal values of $\sigma_{x x}^{c}$ but using a different approach. They were restricted to potentials with ranges less than the magnetic length. For example in a field of 9 tesla (such as that for the IQH transition discussed here) the magnetic length is about $8 \mathrm{~nm}$ whereas the range of the potential in clean,modulation doped, 2DEGs is roughly twice the set-back distance, typically more than $80 \mathrm{~nm}$.

In summary, an integer quantum Hall effect transition measured in a relatively high mobility 2-D system is found to conform rather closely to theoretical predictions. A scattering parameter is extracted which is exponentially dependent on the filling factor and which has a very high degree of symmetry about the critical point. Application of the same formalism to Quantum Hall Hall insulator transitions explains, naturally, the dualities observed there and also emphasises similarities to the $\mathrm{B}=0$ metal insulator transition. It is suggested that failure to observe this canonical behaviour in most Quantum Hall transitions is associated with the conductivities only revealing the underlying properties of the phase transition if the scattering is dominated by short range potentials.

The author would like to thank Dr. R. Williams for material growth, Dr. Y. Feng for sample preparation and Dr. A.S. Sachrajda and P. Zawadzki for use of the dilution refrigerator and for assistance with the measurements. Also Professors S. Das Sarma, D.-H. Lee and S.L. Sondhi for helpful discussions.
${ }^{1}$ S. Das Sarma, in "Perspectives in Quantum Hall Effects", eds S. Das Sarma and A. Pinczuk, John Wiley and Sons (1997), p 1.

2 S.L. Sondhi, S.M. Girvin, J.P. Carini and D. Shahar, Rev. Mod. Phys. 69315 (1997)

${ }^{3}$ Y. Huo, R.E. Hetzel and R.N. Bhatt, Phys. Rev. Lett. 70, $481(1993)$

${ }^{4}$ D.H. Lee, Z. Wang and S. Kivelson, Phys. Rev. Lett. 70, 4130 (1993); S. Kivelson, D.-H. Lee and S.-C. Zhang, Phys. Rev. B 46, 2223 (1992)

${ }^{5}$ B. Huckestein, Rev. Mod. Phys. 67, 357 (1995) and references therein

${ }^{6}$ I. Ruzin and S. Feng, Phys. Rev. Lett. 74, 154 (1995)

${ }^{7}$ H.P. Wei, D.C. Tsui, M.A. Paalanen and A.M.M. Pruisken, Phys. Rev. Lett. 61, 1294 (1988)

${ }^{8}$ D. Shahar, D.C. Tsui, M. Shayegan, R.N. Bhatt and J.E. Cunningham, Phys. Rev. Lett. 74, 4511 (1995)

${ }^{9}$ L.W. Wong, H.W. Jiang, N. Trivedi and E. Palm, Phys. Rev. B 51, 18033 (1995)

10 Sample CVD124 described in "Proceedings of COMMAD1996" ed. C. Jagadish, IEEE N.J. (1997), p414; also sample A in Ref. 11.

${ }^{11}$ P.T. Coleridge, A.S. Sachrajda, P. Zawadzki, R.L Williams and H. Lafontaine, Sol. St. Comm. 102, 755 (1997)

${ }^{12}$ As the field is increased, however, the $(1 \uparrow)$ and $(0 \downarrow)$ levels approach because the exchange enhancement, relative tothe cyclotron spacing, is reduced. In similar, but less dense samples, the reduction is sufficient to cause the two levels to actually overlap near $\nu=1.5$. The small deviations near $\sigma_{x y}=1$, observed in fig $2 \mathrm{~b}$, can probably be associated with this phenomenon.

${ }^{13}$ V. Dobrosavljevic, E. Abrahams, E. Miranda and S. Chakravarty, Phys. Rev. Lett.79, 455 (1997)

14 S.V. Kravchenko, D. Simonian, M.P. Sarachik, W. Mason and J.E. Furneaux, Phys. Rev. Lett. 77, 4938 (1996)

${ }^{15}$ D. Simonian, S.V. Kravchenko and M.P. Sarachik, Phys. Rev. B 77, R13421 (1997)

${ }^{16}$ D. Shahar, M. Hilke, C.C. Li, D.C. Tsui, S.L.Sondhi,J.E. Cunningham and M. Razeghi, Sol. St. Comm. 107, 19 (1998)

17 D. Shahar, D.C. Tsui, M. Shayegan, E. Shimshoni and S.L. Sondhi, Phys. Rev. Lett. 79, 479 (1997)

${ }^{18}$ D. Shahar, D.C. Tsui, M. Shayegan, E. Shimshoni and S.L. Sondhi, Science 274, 589 (1996)

${ }^{19}$ It should be noted that over the temperature range for which a single crossing point is observed, the temperature dependence seen in Fig. 1 which is explained by scaling with $\kappa=3 / 7$, is also consistent (within experimental error) with a linear $\mathrm{T}$ dependence like that observed in Ref. 16.

${ }^{20}$ P.T. Coleridge, P. Zawadzki and A.S. Sachrajda, Phys. Rev. B 49, 10798 (1994)

${ }^{21}$ T. Ando and Y. Uemura, J. Phys. Japan 36,959 (1974)

22 J.T. Chalker and P.D. Coddington, J. Phys. C21,2665 (1988) 


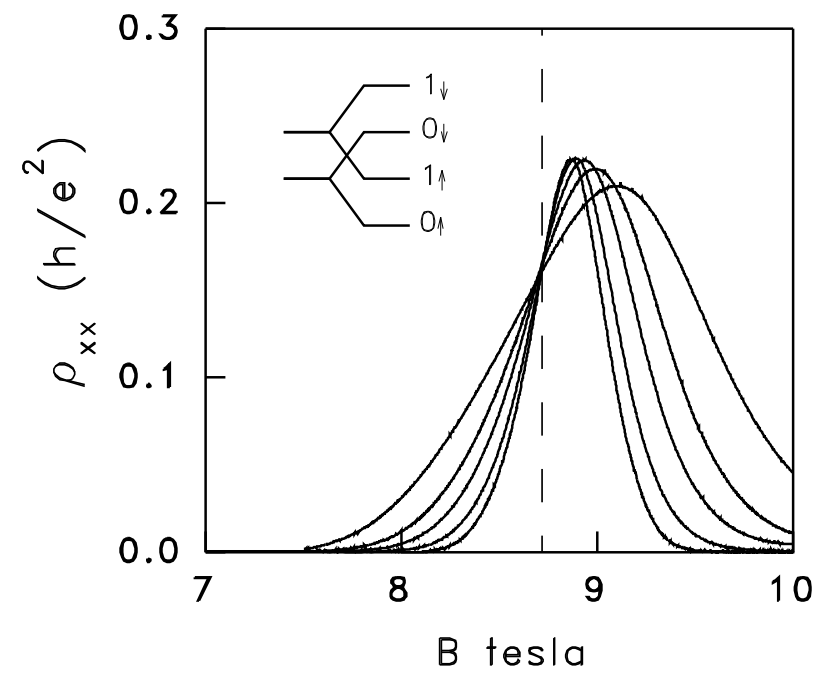

FIG. 1. Resistivity data $\left(\rho_{x x}\right)$ for the $\nu=2$ to 1 IQH effect transition at temperatures of 70,120,180,250 and $400 \mathrm{mK}$. The inset shows, schematically the ferromagnetic alignment of the spins at $\nu=2$.
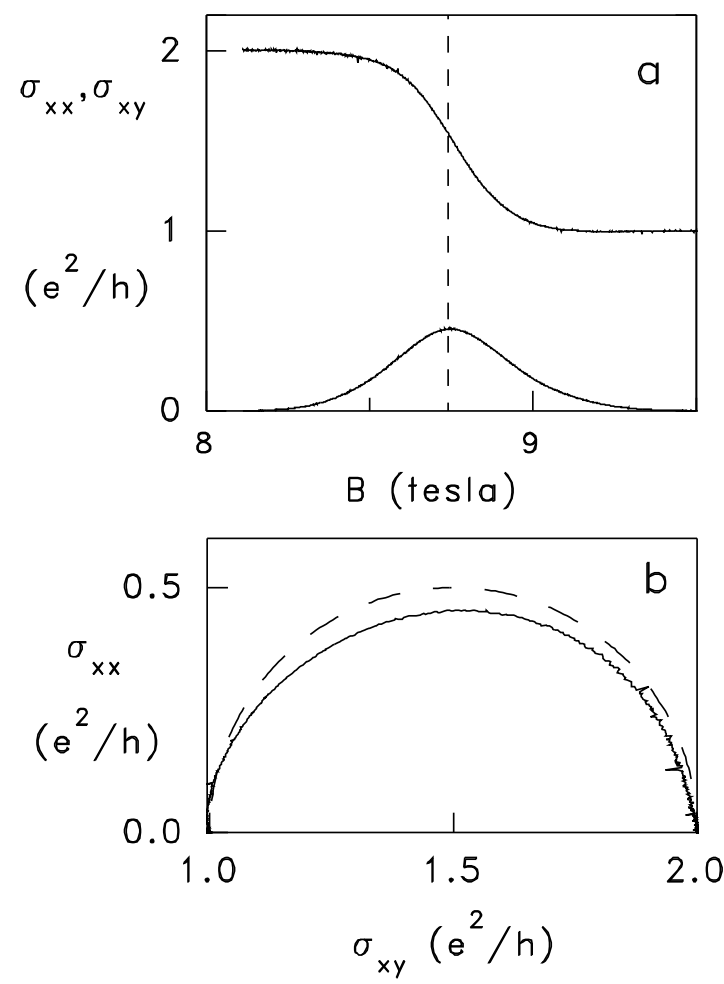

FIG. 2. (a) Conductivity data obtained by inverting resistivities measured at approximately $65 \mathrm{mK}$. The dashed line shows the critical field indicated in figure 1 . (b) $\sigma_{x x}$ plotted against $\sigma_{x y}$ with the semicircular relationship, $\sigma_{x x}^{2}+\left(\sigma_{x y}-3 / 2\right)^{2}=1 / 4$ shown, dashed,for comparison.

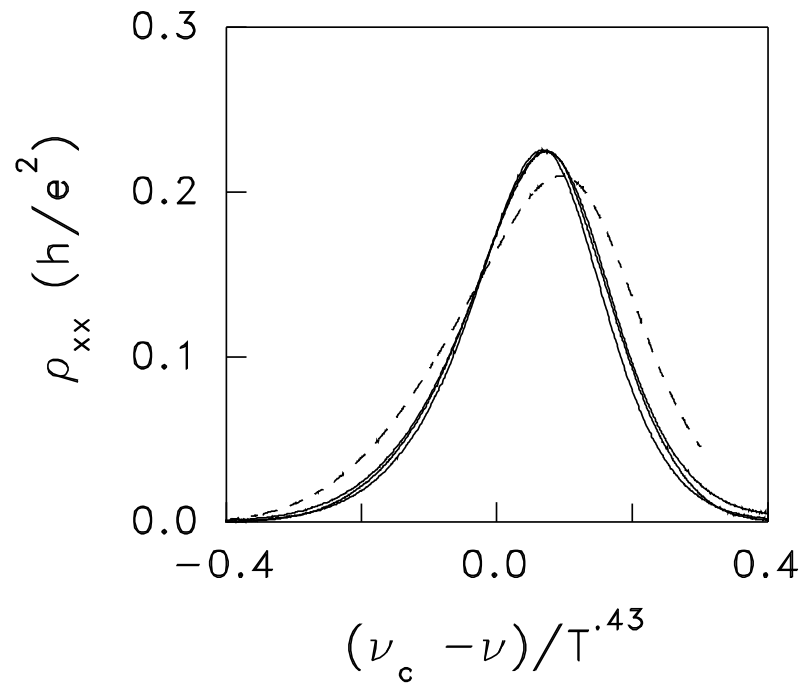

FIG. 3. Scaling curves showing the resistivity data from fig. 1 , for $\mathrm{T}=70,120,180 \mathrm{mK}$, plotted against $\left(\nu_{c}-\nu\right) / T^{\kappa}$ with $\mathrm{T}$ in kelvins, $\kappa=3 / 7$ and $\nu_{c}$, determined from fig. 1 , equal to 1.612. The dashed line is $400 \mathrm{mK}$ data.

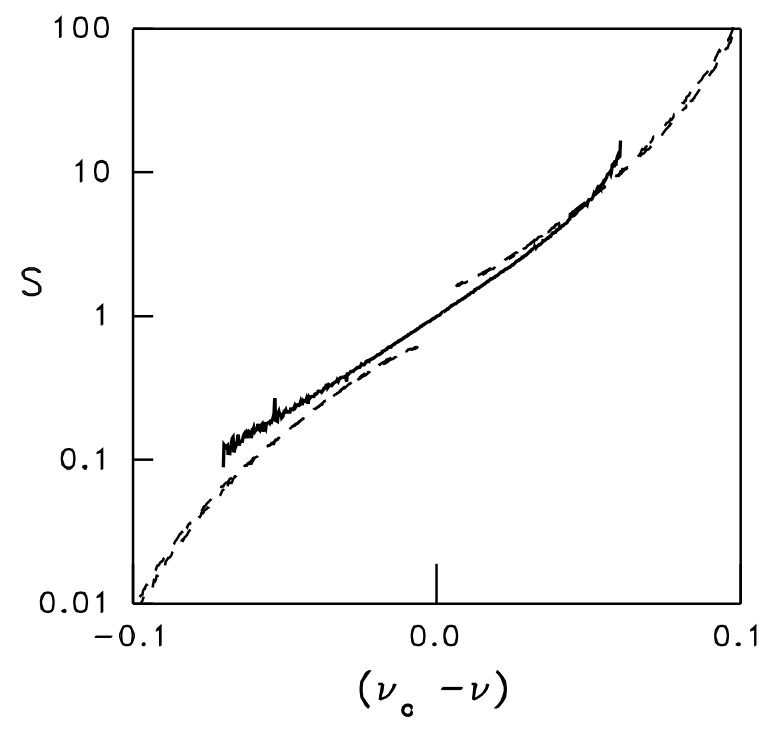

FIG. 4. Scattering parameter s, as defined in the text, determined from the components of the conductivity plotted on a logarithmic scale. Solid line, derived from $\sigma_{x y}$; dashed line, derived from $\sigma_{x x}$, plotted both as s against $\delta \nu$ and as $1 / \mathrm{s}$ against $-\delta \nu$. 